\title{
Host Cell Phenotypic Variability Induced by Trypanosomatid-Parasite-Released Immunomodulatory Factors: Physiopathological Implications
}

\author{
Ali Ouaissi, ${ }^{1 *}$ Mehdi Ouaissi, ${ }^{2}$ Joana Tavares, ${ }^{3}$ and Anabela Cordeiro-Da-Silva ${ }^{3,4}$ \\ ${ }^{1}$ Institut de la Recherche pour le Développement, Unité de Recherche no 008 "Pathogénie des Trypanosomatidae," Montpellier, France \\ ${ }^{2}$ Service de Chirurgie Digestive et Générale, Hôpital Sainte Marguerite, 270 Boulevard de Sainte Marguerite, Marseille, France \\ ${ }^{3}$ Biochemical Laboratory, Faculty of Pharmacy, University of Porto, Portugal \\ ${ }^{4}$ Institute of Molecular and Cellular Biology, University of Porto, Portugal
}

Received 6 November 2003; revised 27 January 2004; accepted 11 February 2004

\begin{abstract}
The parasitic protozoa Trypanosoma cruzi and Leishmania sp release a variety of molecules into their mammalian hosts (ESA: excretory-secretory products). The effects of these ESA on the host cell function may participate in the establishment of a successful infection, in which the parasite persists for a sufficient period of time to complete its life cycle. A number of regulatory components or processes originating from the parasite that control or regulate the metabolism and the growth of host cell have been identified. The purpose of the present review is to analyze some of the current data related to the parasite ESA that interfere with the host cell physiology. Special attention is given to members of conserved protein families demonstrating remarkable diversity and plasticity of function (ie, glutathione S-transferases and related molecules; members of the trans-sialidase and mucin family; and members of the ribosomal protein family). The identification of parasite target molecules and the elucidation of their mode of action toward the host cell represents a step forward in efforts aimed at an immunotherapeutic or pharmacological control of parasitic infection.
\end{abstract}

\section{INTRODUCTION}

Trypanosoma cruzi, the etiological agent of Chagas' disease, is an obligate intracellular parasite causing chronic infections in human and a large number of other mammalian species [1]. This protozoan parasite is transmitted to man and other vertebrate hosts in the faeces of haematophagous bugs of the Reduviid family. The complex life cycle of $T$ cruzi includes different stages in the insect vector and the vertebrate host. There are two parasite stages in the vector: epimastigotes and metacyclic trypomastigotes, whereas the vertebrate stages are bloodstream trypomastigotes and intracellular amastigotes. Chagas' disease is associated with many immunological and immunopathological reactions. In fact, during either the chronic or the acute phase, various autoimmune phenomena were observed and could be the results of nonspecific polyclonal activation or suppressive effects that occur during $T$ cruzi infection. The existence of antigens cross-reactive between $T$ cruzi and mammalian cells was also reported as a possible cause of exacerbation of pathological manifestations [1].

Considerable progress has been made during the past few years on the role of certain parasite and host cell gly- coconjugates, such as glycolipids and glycoproteins, in the process of host cell invasion leading to a proposed model of host cell invasion by $T$ cruzi modulated by positive and negative controls [2]. The positive control is the sialoglycoprotein whereas the negative control is exerted by the neuraminidase, which promotes the desialylation, and thus decreases the level of infection.

Leishmaniasis is a significant cause of morbidity and mortality in several countries worldwide. The disease is caused by a group of kinetoplastid protozoan parasites transmitted by a blood-feeding dipteran vector of the subfamily phlebotominae. Within the insect vector, the parasite replicates as noninfective promastigote, which transforms into an infective metacyclic one. In the mammalian host, the infective promastigotes invade the macrophages and differentiate into amastigotes which are the proliferate forms within the vertebrate host. The subsequent success of a Leishmania infection is dependent on the ability of the parasite, initially in the promastigote form and later in the amastigote one to adhere specifically to, to enter macrophages and to survive within an antigen-presenting cell that has evolved to kill invading microbes.

Leishmaniasis is difficult to treat (specially in AIDS patients). Until vaccines become available, conventional 
measures such as epidemiological surveillance including reservoir control are among the practical options for prevention and containment of the disease. Among the possibilities for development of vaccine against Leishmania, immunization with live, albeit attenuated, organisms has the advantage of mimicking natural infection, still the most effective form of inducing resistance in Leishmaniasis. A detailed investigation of the biology of the parasite has been advocated by the International Health Organization with the hope that it leads to the discovery of more efficient means to combat infection [3]. Particularly, the identification and study of the factors that promote virulence of the parasite may provide new and adequate targets for chemo- or immunotherapy.

The invasion of the host macrophage and tissues depends upon complex processes including ligand-receptor interactions and the production of proteases, which degrade extracellular matrix components $[4,5]$. Much work has focused on the identification of promastigote and macrophage surface molecules involved in the recognition processes. The two major surface glycoconjugates, lipophosphoglycan (LPG) and gp63 have been shown to play a key role in the attachment of promastigotes to macrophages (reviewed in [6]). The macrophage complement receptors (CR3 and CR1), the mannose-fucose receptor, the receptor for advanced glycosylation end products, and the fibronectin receptors were found to play a role in the parasite-cell adhesion phenomenon $[7,8,9$, $10,11]$. Like many other parasitic protozoa, Leishmania preadapts itself to survival in the next phase of its life cycle. Indeed, the differentiation of promastigotes into intracellular amastigotes correlates with their ability to develop means by which they could survive environmental extremes such as toxic metabolites and acidic $\mathrm{pH}$ of the host cell phagolysosomes $[12,13,14,15]$, allowing them to establish infections in mammalian hosts. Studies on the basic cellular function of these organisms led to the identification of several membrane proteins and enzymes, which are essential for the parasite survival in its hosts [16]. However, investigations are still in progress to better understand the molecular basis of virulence in Leishmania parasites. Molecules essential to amastigotes could constitute the basis to engineer successful attenuated life vaccines as they would allow initiation of the infection with the consequent stimulation of the immune system with native antigens but would prevent establishment of productive infection. Different attenuated lines of Leishmania were indeed shown to confer protection in animal models, but their use as vaccines is unacceptable because reversion to virulence cannot be dismissed with confidence. The recent ability to manipulate the genome of Leishmania through gene targeting provides a powerful means to engineer strains of Leishmania which are nonvirulent due to selective mutations or replacements and, therefore, can be safely used as immunogens. The production of a Leishmania major dihydrofolate reductase thymidilate synthase (DHFR/TS) knockout strain auxotrophic for thymidine, illustrates the potential of this approach for Leishmania vaccination [17]. Although unable of in vivo replication, this strain persisted in macrophages time enough for an immune response to develop. This response was able to protect mice against challenge with virulent parasites. Therefore, experimentally generated genetically modified parasite clones exhibiting various biological phenotypes have been used to analyze Leishmania virulence factors [18]. In more recent elegant experiments, such approach has been used to define parasite factors affecting the persistence of the pathogen in its vertebrate host and their role in disease progression [19].

Moreover, in recent study, we found that a previously described gene ( LmSIR2) encoding a protein bearing domain structure characteristic of SIR2 (silent information regulatory 2) proteins [20] when overexpressed in Leishmania infantum amastigotes led to parasite life span extension [21], suggesting therefore its implication in the physiological processes that control parasite survival and development. Studies are in progress in our laboratory to generate parasites with a deficiency state of SIR2-related protein by gene targeting and to analyze their virulence both in vitro and in vivo (Vergnes, Sereno, and Ouaissi, in preparation).

\section{PARASITE-RELEASED FACTORS WHICH BLOCK THE EXPRESSION OF POTENTIALLY HOST-PROTECTIVE INFLAMMATORY RESPONSES}

$T$ cruzi and Leishmania sp produce a number of pathogenic features in humans and different strains of mice and offer the opportunity for studying parameters involved in parasitic infections and disease development. One interesting feature, which has concerned us, is the phenomenon of immunosuppression, a property shared with many other parasites [22]. T cruzi- and Leishmania $s p$-mediated immunosuppression has been documented by several investigators who have used a variety of immune responses to foreign antigens and mitogens, mixed lymphocyte reactions; and skin graft survival [23]. The effect is transient, however, and infected animals eventually produce cell-mediated and humoral responses against the pathogen. A role has been proposed for the involvement of immunosuppressive excreted-secreted antigens (ESA), which serve to maintain chronic infections.

In recent years, we have examined the mechanism(s) of action of a number of T cruzi and Leishmania sp ESA found to act as regulatory factors (activation/inhibition) of host immune cells (ie, $\mathrm{T}$ and/or B cells, macrophages, dendritic cells) $[24,25,26]$. Among these parasitereleased factors, molecules belonging to the glutathione S-transferases (GSTs) super family seem to play a role. The GSTs are isoenzymes playing a variety of redoxrelated roles in organisms ranging from Escherichia coli to man [27]. They are well-characterized proteins with conserved active site found mainly within their N-terminal region also named glutathione binding site (G-site). The 
nonspecific hydrophobic C-terminal region (H-site), which accommodates the electrophilic substrate, has a much more variability than the G-site. A number of GSTrelated molecules from pathogenic human parasites have been shown to function as immunomodulatory factors toward the cells of the immune system [28]. Indeed, in the case of $T$ cruzi, we have previously identified a protein of molecular mass $52 \mathrm{kd}$ whose primary sequence showed two homologous domains sharing significant similarities to GSTs mainly within a region at their N-terminus [29]. Interestingly, the Tc52 was found to be able to catalyze the thiol-disulfide exchange between dihydrotrypanothione and glutathione disulfide and as such was considered as a trypanothione-glutathione thioltransferase [30, 31].

In addition to its enzymatic activity, Tc52 is released by the parasites and was shown to have several cytokine and chemokine-like activities being able to synergize with IFN- $\gamma$ to stimulate NO production by macrophages and to modulate IL-1 $\alpha$, IL-12, and IL-10 encoding genes. These observations may have some implications in vivo. Indeed, we showed that elevated levels of circulating Tc52 in the blood of T cruzi experimentally infected mice occurred during the acute phase of the disease and were associated with decreased responsiveness of $\mathrm{T}$ cells to mitogen or anti-CD3 stimulation [32]. However, it is interesting to remind that Chagas' disease almost exclusively involves intracellular amastigotes, which also expressed Tc52 protein. Therefore, we examined the effect of intracellular production of Tc52 on cell physiology. Transfection experiments with a mammalian expression pcDNA3 plasmid carrying the Tc52 gene, using macrophages and fibroblasts, two type of cells known to harbor $T$ cruzi amastigotes, showed increased IL-10 mRNA levels in the case of macrophages whereas increased transcription of genes encoding GM-CSF and TNF- $\alpha$ was observed in the case of L929 fibroblast cells (reviewed in [28]). Furthermore, in a recent study [33], using single mutant parasite clones $\left(T c 52^{+-}\right)$we analyzed the immune response and the disease phenotype in $T c 52^{+/-}$-infected BALB/c mice, during the acute and chronic phases of the disease. Interestingly, a lack of suppression of IL-2 production and of T-cell proliferation inhibition was observed in the case of spleen cells from $T c 52^{+/-}$-infected mice when compared to wild-type (WT) parasite-infected ones. Moreover, increased production of IL-10 was observed in the case of spleen cells from WT-infected mice, whereas the levels observed in the case of $T c 52^{+/-}$-infected mice were comparable to those of normal mice spleen cells, suggesting therefore, that Tc52 plays a role in IL- 10 cytokine regulation during in vivo T cruzi infection. It is noteworthy that murine IL-10 can downregulate the host immune response by decreasing the production of IL-2 [34] and inhibiting mitogen driven T-cell proliferation [35]. Therefore, it is reasonable to suggest that the reduction of Tc52 production by gene targeting which in turn downregulates the IL-10 synthesis could be among the mechanisms participating in the immunregulatory mechanisms leading to the control of IL-2 production.
Dendritic cells (DC) play a central role in the initiation of immune responses. A recent interesting and informative review has summarized the recent developments of DC-parasite interplay [36]. In order to gain insights into the biological activity toward these cells, we conducted experiments to investigate the Tc52-DC interactions [26]. We show that Tc52 induces human DC maturation via Toll-like receptor 2 (TLR2). Upon incubation with Tc52, DC acquire CD83 and CD86 expression, produce inflammatory chemokines (IL-8, MCP-1, and MIP$1 \alpha$ ), and present potent costimulatory properties. Surprisingly, while the binding of Tc52 to DC was a saturable process, the Tc52-mediated signaling involves its GSHbinding site, whereas another portion of the molecule is involved in Tc52 binding to DC, suggesting the existence of complex molecular interactions (receptors cooperation, spatial organization of recognition structures on the parasite surface?) between the T cruzi-derived molecule, Tc52, and DC. Others have reported that the parasite trans-sialidase (TS) activates mouse DC at least partly via CD43 ligation [37].

The glycoinositolphospholipids (GIPLs) from $T$ cruzi have also been the subject of numerous investigations. Indeed, recent studies have shown that glycosylphosphatidylinositol (GPI) anchors and GIPLs from $T$ cruzi are potent activators of human and mouse macrophage TLR2 [38]. Interestingly, the TLR2 activation by GPI led to the synthesis of IL-12 and TNF- $\alpha$, a profil similar to that observed when using LPS as a triggering agent. In contrast, TLR 2 activation by Tc52 resulted in IL-8, MCP-1, and MIP- $1 \alpha$ production, whereas no secretion of TNF- $\alpha$ occurred. Altogether, these observations suggest that the parasite secrete molecules, which upon interaction with the host cell may trigger signaling pathways leading to different patterns of cell activation/inhibition.

In addition to its role as a virulence factor, Tc52 under conditions of experimental infections appears immunologically relatively silent during the early acute phase, failing to elicit significant levels of antibodies and lymphocyte proliferation. We have hypothesized that the analysis of structure-function relationship in the Tc52 molecule could reveal discrete domains, which might minimize its antigenicity. Studies recently reported identified a naturally occurring major peptide fragment of $28 \mathrm{kd}$ molecular mass (Tc28k) localized in the carboxy-terminal portion of Tc52 carrying the inhibitory capacity on T-cell activation. Synthetic peptides spanning the amino-terminal or carboxy-terminal domain of Tc52 protein indicated that the activity is mapped to Tc52 residues 432-445. Moreover, the peptide when coupled to a carrier protein, exhibited increased inhibitory activity on T lymphocyte activation. Furthermore, the coupled peptide significantly down regulated IFN- $\gamma$ and IL-2 secretion. Likewise, in immunized mice, the coupled peptide 432445 was a very poor B- and T-cell antigen compared to the other Tc52 derived peptides. Therefore, the immunomodulatory portion of $T$ cruzi Tc52 virulent factor seems to reside, at least in part, in a conserved sequence 


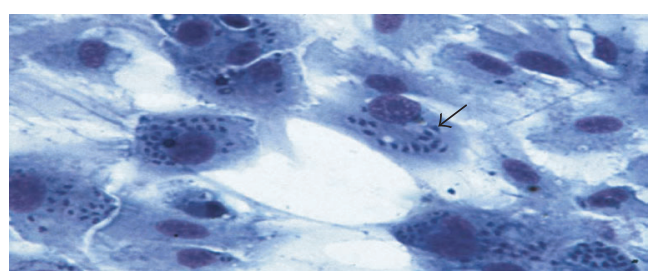

(a)

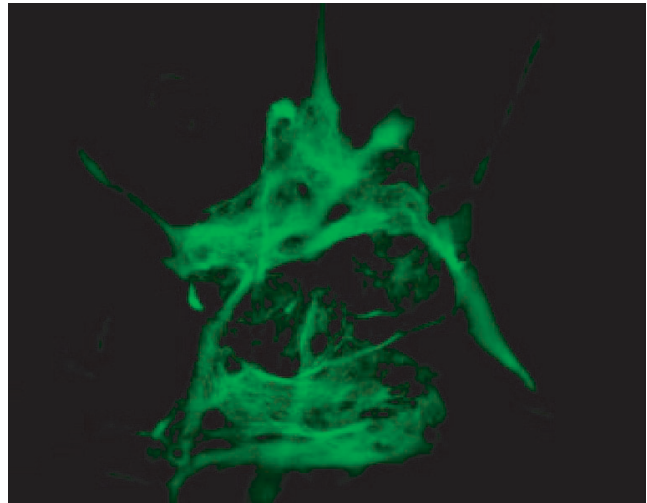

(b)

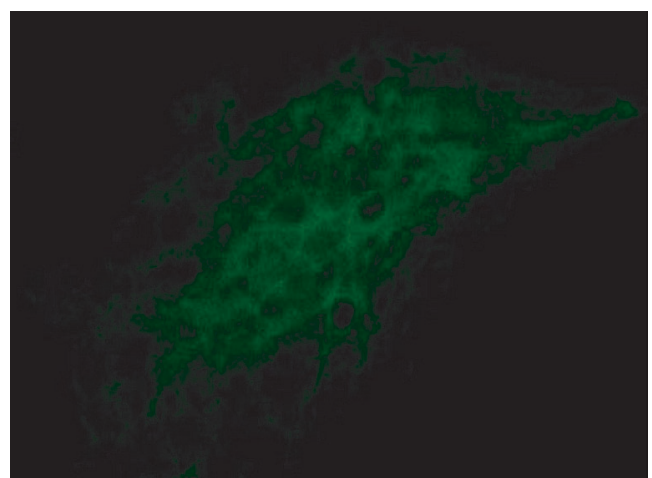

(c)

FIGURE 1. (a) Light microscopy examination of astrocytes infected in vitro by Trypanosoma cruzi trypomastigotes showing highdensity intracellular replicating amastigotes (arrow). (b) and (c) Effect of trypomastigotes upon immunologically detectable glial fibrillary acidic protein (GFAP) of living rat astrocytes. Briefly, primary cultures astrocytes were made from newborn rat cerebral cortex. After the meninges had been cleaned off, the brain tissue was forced gently through a nylon sieve. Dulbecco's modified Eagle medium supplemented with $10 \%$ calf serum, $2 \mathrm{mM}$ glutamine, $50 \mu \mathrm{g} / \mathrm{mL}$ gentamycin was used for the dissociation of cerebral tissue and development of astrocytes. Aliquots $(0.2 \mathrm{~mL})$ of cell suspension containing $2 \times 10^{4}$ cells were placed in 24-well microplates; each well contained a glass coverslip (12 mm diameter). The cells were allowed to attach to the solid phase, and then viable trypomastigotes were added in culture medium at $10^{5}$ parasites per well. After 3 hours at $37^{\circ} \mathrm{C}$, each well was emptied and washed twice to remove free-swimming parasites. The cells were fixed in $0.1 \%$ paraformaldehyde in PBS at $4^{\circ} \mathrm{C}$ during 20 minutes and washed with PBS. They were then incubated for 1 hour at room temperature in PBS containing $1 \%$ triton $\times 100$ and $1 \%$ bovine serum albumin (BSA). After three washes in PBS, the cells were treated with fluorescein-conjugated rabbit anti-GFAP antibodies diluted $1: 100$ in PBS-BSA. After three washes in PBS, the cells were counterstained with $0.02 \%$ Evans blue in PBS and, in order to reduce fading, mounted in Citifluor AF1 (glycerol: PBS solution (9/1), Citifluor Ltd, London,UK). (b) Fluorescence micrograph of astrocytes incubated with culture medium alone and reacted with anti-GFAP antibodies showing normal distribution of GFAP. (c) Cell distribution of GFAP network after infection with trypomastigotes.

within its carboxyl-terminal domain which could minimize its antigenicity [39]. Such molecules may permit parasites to escape immune surveillance and to grow unimpeded by normal immune responses. Moreover, the impairment of multiple immune effector functions by blocking the signal transduction pathways utilized by cytokines such as IL-2 and IFN- $\gamma$; the host may become more susceptible to opportunistic infections as well.

Like other microorganisms, T cruzi and Leishmania $s p$ release a large number of molecules which could act as mitogenic substances inducing polyclonal lymphocyte responses and consequently a general lack of specificity of antibodies and T-cell responses during the infection. The mechanisms by which T cruzi and Leishmania sp activate "panclonal" B lymphocyte response are fundamental, and may contribute to the immune deregulation. A num- ber of $T$ cruzi-released proteins behave as B-cell activators (reviewed in [29]). Soluble parasite-derived antigens from $L$ major and $L$ donovani are mitogenic and trigger the production of immunoglobulins with autoantibody activity [40]. Thus, crude extracts of $L$ donovani and $L$ mexicana amazonensis contain components, which cause strong in vitro polyclonal activation of hamster spleen cells [41]. Moreover, an excreted factor derived from the culture medium of $L$ major was found to suppress Con A induced polyclonal activation of mouse T cells [42]. Moreover, we have identified a parasite gene encoding a protein sharing significant homology to mammalian ribosomal protein S3a named LmS3a exhibiting dual activity being stimulatory and inhibitory toward $\mathrm{T}$ and $\mathrm{B}$ cells, respectively [25]. Analysis of cytokine production revealed a significant downregulation of IFN- $\gamma$, IL-2, and IL-12 
secretion by LmS3a. Since induction of Th1 immune response and simultaneous lack of activation of a Th2 response are required for generation of immunity to Leishmaniasis, LmS3a could be among candidate molecules to be tested for optimal design of vaccines against Leishmania parasites.

\section{MODULATION OF HOST CELL DEATH/SURVIVAL BY PARASITES AND THEIR RELEASED FACTORS}

Cell death with cell division generates the proper number and type of cells during development and maintains the balance between different cell populations in an organism. Alteration of this equilibrium by invading parasites results in the development of immunopathological processes and disease progression. The invading parasites can release factors, which kill the cells of the immune system by activating the cellular death machinery, thus inducing apoptosis. For example, one of the major surface glycoconjugate in T cruzi, the GIPL has been shown to induce apoptosis of macrophages through its lipid ceramide domain [43].

Many efforts in the last years have focused on the study of an important family of proteins from $T$ cruzi: TS and mucin families. T cruzi is unable to synthesize the de novo monosaccharide sialic acid, but can incorporate sialic acid derived from the host. This latter function is devoted to the TS, which catalyzes the transfer of sialic acid from host glycoconjugates to mucin-like molecules located on the parasite surface membrane. TS is released by the parasite into the external milieu as a soluble factor, being a modified sialidase able to transfer sialic acid from sialoglycoconjugates from the host to $\beta$-galactoses in the glycoconjugates of the parasite. TS is expressed in the invasive trypomastigote stage and is defined by two regions: a globular amino-terminus of about 640 amino acids containing the catalytic activity and a variable number of a repeated highly antigenic motif of 12 amino acids named SAPA located at the C terminus [44]. It has been shown that under certain circumstances TS could act as an apoptosis inducer in cells of the immune system in vivo [45]. Furthermore, evidences reported support that TS is a virulence factor responsible for thymic alterations via apoptosis of "nurse cell complex" [46].

Paradoxically, a large number of experimental evidences have shown that parasites may inhibit apoptosis of host cells. For instance, we have observed that intracellular production of native Tc52 stimulated the growth of macrophages and fibroblasts (reviewed in [28]). This kind of intriguing biological activity is not unique to the Tc52. In fact, a number of other studies have shown that parasite-derived molecules could interfere with the growth of host cells. In the case of $T$ cruzi, strong evidence indicates that the parasite trans-sialidase (TS) synergizes with the cytokine ciliary neurotropic factor and leukemia inhibitory factor to prevent apoptosis of neuronal cells [47]. TS also triggered $b c l-2$ gene expression leading to
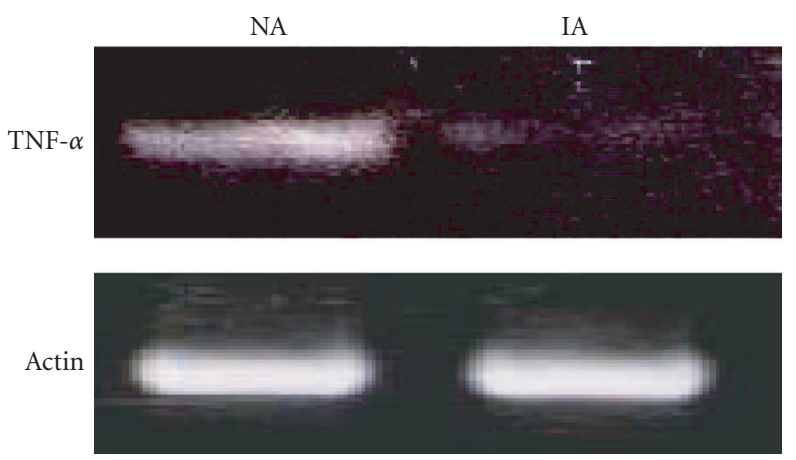

FIgURE 2. Effect of T cruzi infection on TNF- $\alpha$ gene expression in astrocytes. RT-PCR analysis was performed as described in [24]. Briefly, one microgram of total RNA from either infected (IA) or noninfected astrocytes (NA) was reverse-transcribed to cDNA. The cDNA sample was used as a template for 25-35 cycles of PCR using primers for rat TNF- $\alpha$ and actin as a control housekeeping gene to check the homogeneity of different samples. Due to the fact that RNA from infected cells contained an additional source of RNA originating from $T$ cruzi, the preparations were adjusted so that the levels of actin transcripts would be comparable in IA and NA cells.

the protection of rat pheochromocytoma PC12 cells, a cell line that exhibits several characteristics of neurons, against apoptosis induced by growth factor deprivation. Furthermore, TS has been reported to act as a survival factor for Schwann cells by protecting them from apoptosis [48]. In fact, the TS activates Schwann-cell phosphatidylinositol 3-kinase (P13K)/Akt protein kinase signaling, a cell survival pathway.

Moreover, it has been shown that macrophages upon infection became resistant to apoptosis induced by growth factors removal [49]. Since treatment of bone marrowderived macrophages with exogenous $L$ donovani promastigote surface lipophosphoglycan inhibits apoptosis, it has been proposed that the lipophosphoglycan might be involved in the pathway leading to the apoptosis inhibition. This phenomenon seems to be correlated to increased transcription of macrophage cytokine genes encoding GM-CSF, TNF- $\alpha$, TGF- $\beta$, and IL-6. More recently, it has been reported that coincubation of polymorphonuclear neutrophil granulocytes (PMN) with $L$ major promastigotes resulted in significant inhibition of PMN apoptosis. This phenomenon is associated with a significant reduction of PMN caspase-3 activity. Since, parasites were found inside mice skin PMN after subcutaneous challenge infection, it has been suggested that infection with $L$ major prolongs the survival time of PMN in vivo [50]. Taken together, these observations suggest the deployment by the parasites of active strategies that manipulate the host response.

The modulation of host cell metabolism by $T$ cruzi has also been reported (reviewed in [51]). For example, myoblasts infected with $T$ cruzi do not differentiate to myotubes and do not synthesize mRNA for 


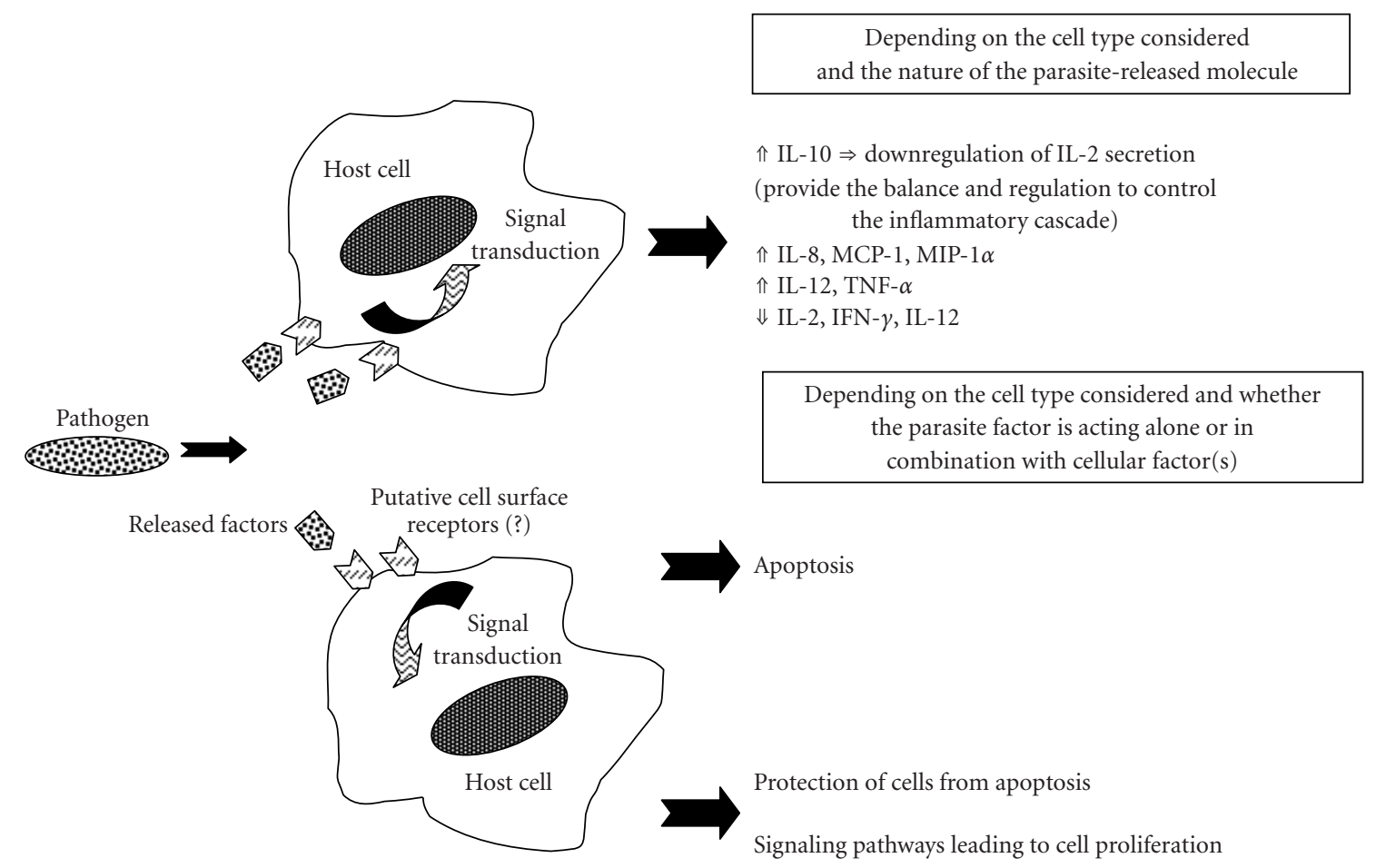

FIGURE 3. Schematic diagrams showing possible pathways by which the parasite-released factors affect the immune cells.

myosin heavy chain and $\alpha$-actin, despite the fact that they produce normal levels of housekeeping mRNA. Moreover, evidence for functional disturbances in the generation of second messenger cAMP has been reported. Furthermore, the infection influences the cytosolic levels of free $\mathrm{Ca}^{2+}$ and the metabolism of inositol phosphates (IP1, IP2, and IP3) as well as the synthesis of the extracellular matrix components.

Central nervous system (CNS) involvement in Chagas' disease has been considered to give a grave prognosis, with fatal outcome in a high percentage of children in the acute phase of the disease. The CNS can be invaded and damaged by a variety of microbes. Studies have shown that astrocytes, microglial resident cells of the CNS, play a key role in CNS defense against infections [52]. The astroglial cytokines are among factors which may help to limit the spread of pathogens through their effect on the recruitment and activity of immune cells. Astrocytes respond vigorously to infections of the CNS; the responses being generally detectable 24 hours after infection. Reactive astrocytosis includes an increase in the number and size of cells expressing the glial fibrillary acidic protein (GFAP). Unexpectedly, we found a significant reduction of immunologically detectable GFAP in astrocytes infected with T cruzi when compared to noninfected cells (Figures $1 \mathrm{~b}$ and 1c). Moreover, although reactive astrocytes have been shown to express elevated levels of TNF- $\alpha$, this seems not to be the case upon infection of astrocytes with $T$ cruzi. Indeed, a significant downregulation of TNF- $\alpha$ gene transcription could be seen in the case of $T$ cruzi-infected astrocytes when compared to noninfected cells (Figure 2). Astrocytes are found throughout the CNS and are in intimate contact with the endothelial cells. Therefore, they could influence the entry of hematogenous cells into the CNS and their activity once within the CNS. The downregulation of astrocyte function by $T$ cruzi might impair the recruitment and activity of immune cells. Thus, the impairment of astrocytes may play a role in the pathogenesis of Chagas' disease particularly in children during the acute phase of T cruzi infection.

\section{CONCLUDING REMARKS}

Parasitic protozoa of the trypanosomatids family and their released products exert a spectrum of influences on the immune cells of their mammalian hosts, ranging from the induction of host-protective immunity to providing mechanisms by which the parasites evade the host's immune surveillance (see Figure 3). However, it is noteworthy that molecules released from parasites in vitro, and designed ESA, may include components, which would not be released from viable parasites in vivo. Therefore, it is of crucial importance to demonstrate, using molecular and immunological approaches, the relevance of the biological activities of ESA molecules in vivo. It is reasonable to suggest that among the released molecules, those, which may confer a selective advantage to the pathogen, represent potential targets for developing therapeutic strategies to blunt the host-immune system dysfunction. 


\section{ACKNOWLEDGMENT}

This work was supported by IRD, INSERM, the Faculty of Pharmacy, University of Porto, and the FrenchPortuguese Scientific and Technological cooperation, project no 706C3.

\section{REFERENCES}

[1] Takle GB, Snary D. South American trypanosomiasis (Chagas' disease). In: Warren KS, Ed. Immunology of Parasitic Infections. Oxford: Blackwell Scientific Publications; 1993:213-236.

[2] Vermelho AB, Meirelles MN. Sialoglycoconjugates in Trypanosoma cruzi-host cell interaction: possible biological models - a review. Mem Inst Oswaldo Cruz. 1994;89(1):69-79.

[3] World Health Organization. Report on the consultative meeting on Leishmania/HIV co-infection. Tech. Rep. WHO/LEISH/95.35, WHO, Geneva, 1995;89:1-14.

[4] Chang KP. Cellular and molecular mechanisms of intracellular symbiosis in leishmaniasis. Int Rev Cytol Suppl. 1983;14:267-305.

[5] Chang KP, Chaudhuri G, Fong D. Molecular determinants of Leishmania virulence. Annu Rev Microbiol. 1990;44:499-529.

[6] Alexander J, Russell DG. The interaction of Leishmania species with macrophages. Adv Parasitol. 1992;31:175-254.

[7] Wilson ME, Pearson RD. Evidence that Leishmania donovani utilizes a mannose receptor on human mononuclear phagocytes to establish intracellular parasitism. J Immunol. 1986;136(12):4681-4688.

[8] Ouaissi A. Role of the RGD sequence in parasite adhesion to host cells. Parasitol Today. 1988;4:169-173.

[9] Ouaissi A, Capron A. Some aspects of protozoan parasite-host cell interactions with special reference to RGD-mediated recognition process. Microb Pathog. 1989;6(1):1-5.

[10] Talamas-Rohana P, Wright SD, Lennartz MR, Russell DG. Lipophosphoglycan from Leishmania mexicana promastigotes binds to members of the CR3, p150,95 and LFA-1 family of leukocyte integrins. J Immunol. 1990;144(12):4817-4824.

[11] Mosser DM, Rosenthal LA. Leishmania-macrophage interactions: multiple receptors, multiple ligands and diverse cellular responses. Semin Cell Biol. 1993;4(5):315-322.

[12] Mukkada AJ, Meade JC, Glaser TA, Bonventre PF. Enhanced metabolism of Leishmania donovani amastigotes at acidic $\mathrm{pH}$ : an adaptation for intracellular growth. Science. 1985;229(4718):1099-1101.

[13] Glaser TA, Baatz JE, Kreishman GP, Mukkada AJ. $\mathrm{pH}$ homeostasis in Leishmania donovani amastigotes and promastigotes. Proc Natl Acad Sci USA. 1988;85(20):7602-7606.

[14] Antoine JC, Prina E, Jouanne C, Bongrand P. Parasitophorous vacuoles of Leishmania amazonensis- infected macrophages maintain an acidic pH. Infect Immun. 1990;58(3):779-787.

[15] Panaro MA, Panunzio M, Jirillo E, Marangi A, Brandonisio O. Parasite escape mechanisms: the role of Leishmania lipophosphoglycan on the human phagocyte functions. A review. Immunopharmacol Immunotoxicol. 1995;17(3):595-605.

[16] Schneider P, Bordier C, Etges R. Membrane proteins and enzymes of Leishmania. Subcell Biochem. 1992;18:39-72.

[17] Titus RG, Gueiros-Filho FJ, de Freitas LA, Beverley SM. Development of a safe live Leishmania vaccine line by gene replacement. Proc Natl Acad Sci USA. 1995;92(22):10267-10271.

[18] Beverley SM. Genetic and genomic approaches to the analysis of Leishmania virulence. In: Marr J, Nilsen T, Komuniecki R, eds. Molecular and Medical Parasitology. New York, NY: Academic Press; 2003:111-122.

[19] Spath GF, Lye LF, Segawa H, Sacks DL, Turco SJ, Beverley SM. Persistence without pathology in phosphoglycan-deficient Leishmania major. Science. 2003;301(5637):1241-1243.

[20] Yahiaoui B, Taibi A, Ouaissi A. A Leishmania major protein with extensive homology to silent information regulator 2 of Saccharomyces cerevisiae. Gene. 1996;169(1):115-118.

[21] Vergnes B, Sereno D, Madjidian-Sereno N, Lemesre JL, Ouaissi A. Cytoplasmic SIR2 homologue overexpression promotes survival of Leishmania parasites by preventing programmed cell death. Gene. 2002;296(1-2):139-150.

[22] Capron A, Dessaint JP. Molecular basis of hostparasite relationship: towards the definition of protective antigens. Immunol Rev. 1989;112:27-48.

[23] Capron A, Camus D. Immunoregulation by parasites extracts. Springer Semin Immunopathol. 1979;2:6977.

[24] Fernandez-Gomez R, Esteban S, Gomez-Corvera R, Zoulika K, Ouaissi A. Trypanosoma cruzi: Tc52 released protein-induced increased expression of nitric oxide synthase and nitric oxide production by macrophages. J Immunol. 1998;160(7):3471-3479.

[25] Cordeiro-Da-Silva A, Borges MC, Guilvard E, Ouaissi A. Dual role of the Leishmania major ribosomal protein S3a homologue in regulation of T- and B-cell activation. Infect Immun. 2001;69(11):65886596.

[26] Ouaissi A, Guilvard E, Delneste Y, et al. The Trypanosoma cruzi Tc52-released protein induces human dendritic cell maturation, signals via Toll-like receptor 2, and confers protection against lethal infection. J Immunol. 2002;168(12):6366-6374.

[27] Neal GE, Moss EJ, Manson MM. Glutathione conjugation in oncogenesis. In: Sies H, Ketterer B, eds. Glutathione Conjugation: Mechanisms and Biological Significance. London: Academic Press; 1988:281314. 
[28] Ouaissi A, Ouaissi M, Sereno D. Glutathione Stransferases and related proteins from pathogenic human parasites behave as immunomodulatory factors. Immunol Lett. 2002;81(3):159-164.

[29] Ouaissi A, Cordeiro-Da-Silva A, Guevara AG, Borges M, Guilvard E. Trypanosoma cruzi-induced host immune system dysfunction: a rationale for parasite immunosuppressive factor(s) encoding gene targeting. J Biomed Biotechnol. 2001;1(1):11-17.

[30] Moutiez M, Aumercier M, Schöneck R, et al. Purification and characterization of a trypanothioneglutathione thioltransferase from Trypanosoma cruzi. Biochem J. 1995;310(pt 2):433-437.

[31] Moutiez M, Quemeneur E, Sergheraert C, Lucas V, Tartar A, Davioud-Charvet E. Glutathionedependent activities of Trypansoma cruzi p52 makes it a new member of the thiol: disulphide oxidoreductase family. Biochem J. 1997;322(pt 1):43-48.

[32] Ouaissi A, Guevara-Espinoza A, Chabé F, GomezCorvera R, Taibi A. A novel and basic mechanism of immunosuppression in Chagas' disease: Trypanosoma cruzi releases in vitro and in vivo a protein which induces $\mathrm{T}$ cell unresponsiveness through specific interaction with cysteine and glutathione. Immunol Lett. 1995;48(3):221-224.

[33] Garzon E, Borges MC, Cordeiro-Da-Silva A, et al. Trypanosoma cruzi carrying a targeted deletion of a Tc52 protein-encoding allele elicits attenuated Chagas' disease in mice. Immunol Lett. 2003;89(1):6780.

[34] Fiorentino DF, Zlotnik A, Vieira P, et al. IL-10 acts on the antigen-presenting cell to inhibit cytokine production by Th1 cells. J Immunol. 1991;146(10): 3444-3451.

[35] Ding L, Shevach EM. IL-10 inhibits mitogeninduced $\mathrm{T}$ cell proliferation by selectively inhibiting macrophage costimulatory function. J Immunol. 1992;148(10):3133-3139.

[36] Sher A, Pearce E, Kaye P. Shaping the immune response to parasites: role of dendritic cells. Curr Opin Immunol. 2003;15(4):421-429.

[37] Todeschini AR, Nunes MP, Previato JO, MendonçaPreviato L, Dosreis GA. Trans-sialidase from Trypanosoma cruzi fully activates host T cells and dendritic cells. Mem Inst Oswaldo Cruz Rio de Janeiro. 2000;95(suppl II):194.

[38] Campos MA, Almeida IC, Takeuchi O, et al. Activation of Toll-like receptor-2 by glycosylphosphatidylinositol anchors from a protozoan parasite. J Immunol. 2001;167(1):416-423.

[39] Borges M, Cordeiro-Da-Silva A, Sereno D, Ouaissi A. Peptide-based analysis of the amino acid sequence important to the immunoregulatory function of Trypanosoma cruzi Tc52 virulence factor. Immunology. 2003;109(1):147-155.

[40] Bohme MW, Evans DA, Miles MA, Holborow EJ. Occurrence of autoantibodies to intermediate filament proteins in human visceral leishmaniasis and their induction by experimental polyclonal Bcell activation. Immunology. 1986;59(4):583-588.

[41] Bunn-Moreno MM, Madeira ED, Miller K, Menezes JA, Campos-Neto A. Hypergammaglobulinaemia in Leishmania donovani infected hamsters: possible association with a polyclonal activator of B cells and with suppression of $\mathrm{T}$ cell function. Clin Exp Immunol. 1985;59(2):427-434.

[42] Grimaldi G Jr, Tesh RB. Leishmaniases of the new world: current concepts and implications for future research. Clin Microbiol Rev. 1993;6(3):230-250.

[43] Freire-de-Lima CG, Nunes MP, Corte-Real S, et al. Proapototic activity of a Trypanaosoma cruzi ceramide-containing glycolipid turned on in host macrophages by IFN- $\gamma$. J Immunol. 1998;161(9): 4909-4916.

[44] Frasch AC. Functional diversity in the trans-sialidase and mucin families in Trypanosoma cruzi. Parasitol Today. 2000;16(7):282-286.

[45] Leguizamon MS, Mocetti E, Garcia Rivello H, Argibay PF, Campetella O. Trans-sialidase from Trypanosoma cruzi induces apoptosis in cells from the immune system in vivo. J Infect Dis. 1999;180(4): 1398-1402.

[46] Mucci J, Hidalgo A, Mocetti E, Argibay PF, Leguizamon MS, Campetella O. Thymocyte depletion in Trypanosoma cruzi infection is mediated by transsialidase-induced apoptosis on nurse cells complex. Proc Natl Acad Sci USA. 2002;99(6):3896-3901.

[47] Chuenkova MV, Pereira MA. A trypanosomal protein synergizes with the cytokines ciliary neurotrophic factor and leukemia inhibitory factor to prevent apoptosis of neuronal cells. Mol Biol Cell. 2000;11(4):1487-1498.

[48] Chuenkova MV, Furnari FB, Cavenee WK, Pereira MA. Trypanosoma cruzi trans-sialidase: a potent and specific survival factor for human Schwann cells by means of phosphatidylinositol 3-kinase/Akt signaling. Proc Natl Acad Sci USA. 2001;98(17):9936-9941.

[49] Moore KJ, Matlashewski G. Intracellular infection by Leishmania donovani inhibits macrophage apoptosis. J Immunol. 1994;152(6):2930-2937.

[50] Aga E, Katschinski DM, van Zandbergen G, et al. Inhibition of the spontaneous apoptosis of neutrophil granulocytes by the intracellular parasite Leishmania major. J Immunol. 2002;169(2):898-905.

[51] Morris SA, Tanowitz HB, Bilezikian JP, Wittner M. Modulation of host cell metabolism by Trypanosoma cruzi. Parasitol Today. 1991;7(4):82-87.

[52] Mucke L, Eddleston M. Astrocytes in infectious and immune-mediated diseases of the central nervous system. FASEB J. 1993;7(13):1226-1232.

\footnotetext{
* Corresponding author.
}

E-mail: ali.ouaissi@montp.inserm.fr

Fax: + 334674163 31; Tel: + 33467416331 


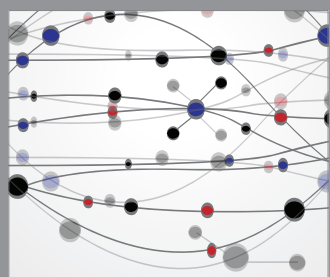

The Scientific World Journal
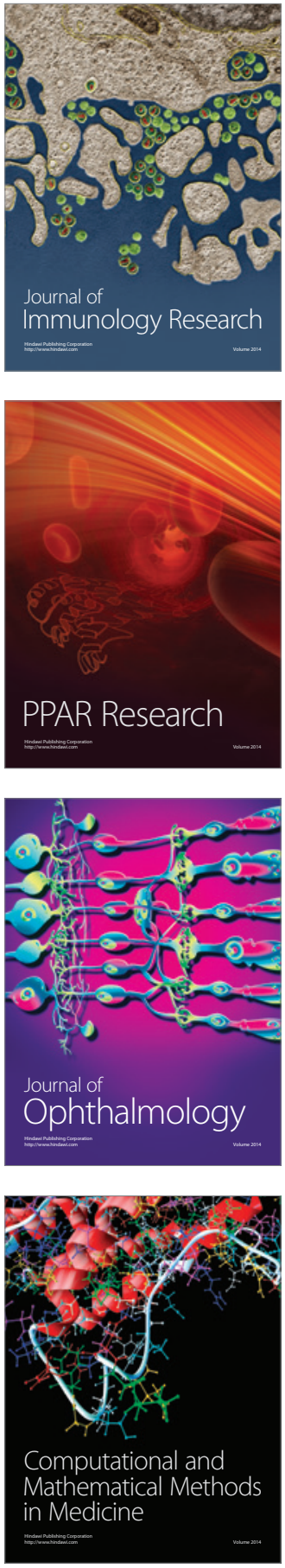

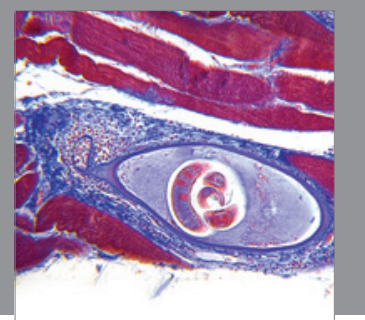

Gastroenterology

Research and Practice
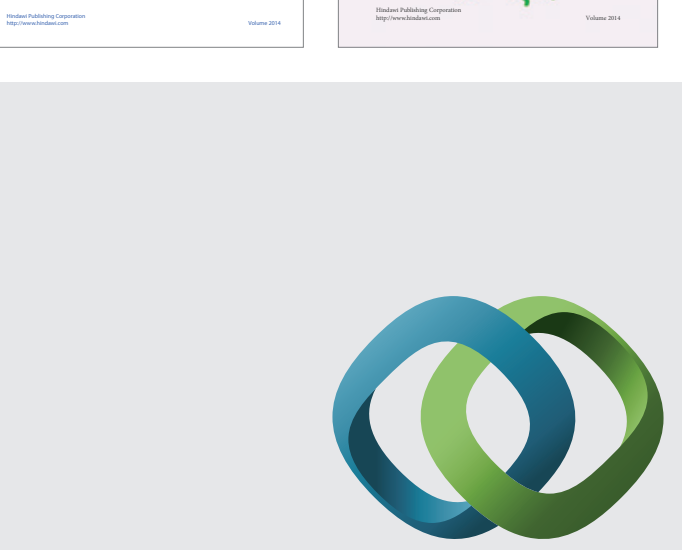

\section{Hindawi}

Submit your manuscripts at

http://www.hindawi.com
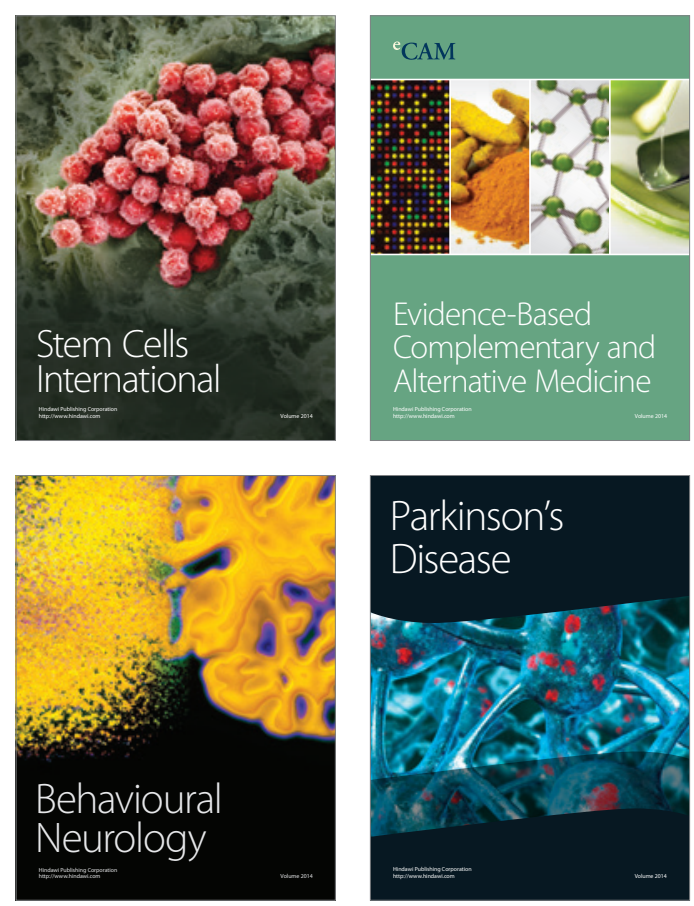

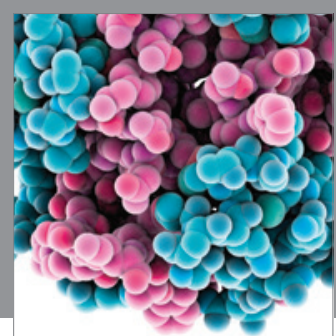

Journal of
Diabetes Research

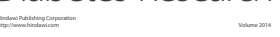

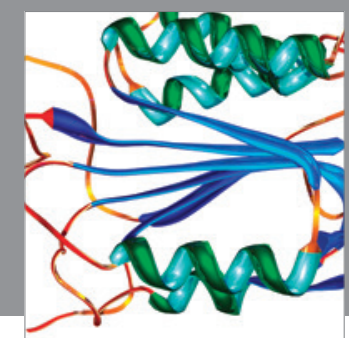

Disease Markers
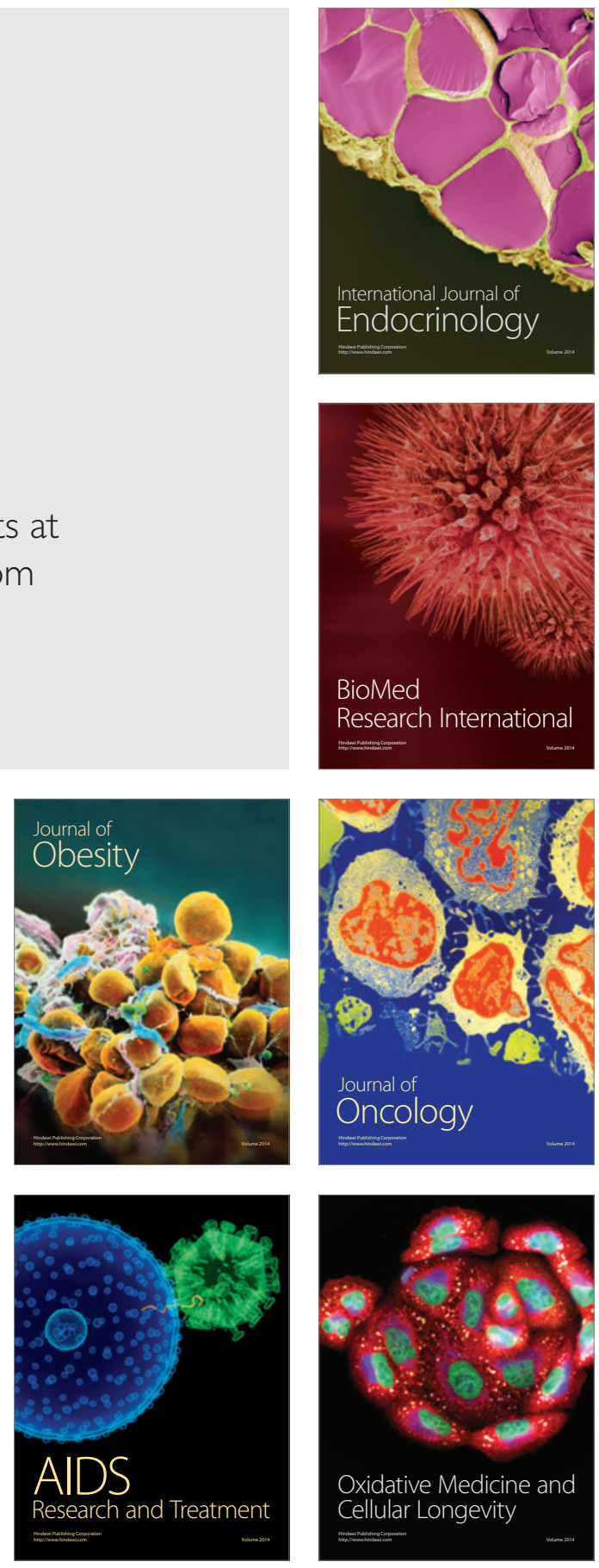